\title{
BMJ Open Collaboration between patient organisations and a clinical research sponsor in a rare disease condition: learnings from a community advisory board and best practice for future collaborations
}

Annelise Roennow, ${ }^{1,2}$ Maureen Sauvé, ${ }^{3}$ Joep Welling (10 , ${ }^{1}$ Robert J Riggs, ${ }^{4}$ Ann Tyrrell Kennedy, ${ }^{1}$ Ilaria Galetti, ${ }^{1,5}$ Edith Brown, ${ }^{6}$ Catarina Leite, ${ }^{7}$ Alex Gonzalez, ${ }^{8}$ Alexandra Paula Portales Guiraud, ${ }^{1,9}$ François Houÿez, ${ }^{10}$ Rob Camp, ${ }^{10}$ Annie Gilbert, ${ }^{11}$ Martina Gahlemann, ${ }^{12}$ Lizette Moros, ${ }^{13}$ Jose Luis Luna Flores, ${ }^{13}$ Friedrich Schmidt, ${ }^{13}$ Wiebke Sauter, ${ }^{14}$ Henrik Finnern ${ }^{13}$

To cite: Roennow A, Sauvé M, Welling J, et al. Collaboration between patient organisations and a clinical research sponsor in a rare disease condition: learnings from a community advisory board and best practice for future

collaborations. BMJ Open 2020;10:e039473. doi:10.1136/ bmjopen-2020-039473

- Prepublication history and additional material for this paper is available online. To view these files, please visit the journal online (http://dx.doi.org/10. 1136/bmjopen-2020-039473).

Received 16 April 2020

Revised 24 September 2020

Accepted 04 November 2020

Check for updates

(c) Author(s) (or their employer(s)) 2020. Re-use permitted under CC BY-NC. No commercial re-use. See rights and permissions. Published by BMJ.

For numbered affiliations see end of article.

Correspondence to Joep Welling;

joep.at.fesca@gmail.com

\section{ABSTRACT}

Introduction Transparent collaborations between patient organisations (POs) and clinical research sponsors (CRS) can identify and address the unmet needs of patients and caregivers. These insights can improve clinical trial participant experience and delivery of medical innovations necessary to advance health outcomes and standards of care. We share our experiences from such a collaboration undertaken surrounding the SENSCIS ${ }^{\circledR}$ clinical trial (NCT02597933), and discuss its impact during, and legacy beyond, the trial.

Summary We describe the establishment of a community advisory board (CAB): a transparent, multiyear collaboration between the scleroderma patient community and a CRS. We present shared learnings from the collaboration, which is split into three main areas: (1) the implementation and conduct of the clinical trial; (2) analysis and dissemination of the results; and (3) aspects of the collaboration not related to the trial.

1. The scleroderma CAB reviewed and provided advice on trial conduct and reporting. This led to the improvement and optimisation of trial procedures; meaningful, patientfocused adaptations were made to address challenges relevant to scleroderma-associated interstitial lung disease patients.

2. To ensure that results of the trial were accessible to lay audiences and patients, written lay summaries were developed by the trial sponsor with valuable input from the $C A B$ to ensure that language and figures were understandable.

3. The $\mathrm{CAB}$ and the CRS also collaborated to co-develop opening tools for medication blister packs and bottles. In addition, to raise disease awareness among physicians, patients and caregivers, educational materials to improve diagnosis and management of scleroderma were co-created and delivered by the CAB and CRS.

Conclusions This collaboration between POs and a CRS, in a rare disease condition, led to meaningful improvements

\section{Strengths and limitations of this study}

- Working in collaboration, patient organisations and clinical research sponsors can co-create and implement initiatives tailored to address the unmet needs of people living with different disease conditions.

- Transparency and recognition of mutual interests from the outset are key to establishing and sustaining effective collaborations.

- Sustaining these collaborations in the long term across the entire medical innovation life cyclefrom inception to clinical trial evaluation and delivery-is imperative to achieve patient-focused improvements in healthcare outcomes.

in patient safety, comfort and self-management and addressed information needs. This collaboration may serve as a template of best practice for future collaborations between $\mathrm{POs}$, research sponsors and other healthcare stakeholders.

\section{PATIENT FOCUS IN DRUG DEVELOPMENT}

The role of patients and caregivers as key stakeholders in clinical research is increasingly recognised by all parties involved in healthcare innovation, regulation and delivery. ${ }^{1-3}$ Patient-focused research is an increasing priority for regulators, ${ }^{4-6}$ health technology assessment (HTA) bodies, payers, ${ }^{7-10}$ pharmaceutical companies and public-private partnerships. ${ }^{11-13}$ For many years, the patient and caregiver community has campaigned for companies to embed patient involvement into all stages of clinical research and development. ${ }^{14} 15$ Patient 
Box 1 Challenges conducting clinical trials in rare diseases

- Poor understanding of disease natural history impedes planning

- Failure to recruit enough patients

- Small number of patients with disease.

- Patients misdiagnosed.

- Restrictive inclusion criteria.

- Competing trials at few centres.

- Physicians outside of expert centres not identifying patients.

- Patients unaware of clinical trials.

- Patients dissuaded by inappropriate clinical trial design.

- Patients dissuaded by the choice of comparator arm.

- Lack of clarity over compassionate use after trial completion.

- Excessive burden of clinic visits and tests for multiple endpoints.

Ineffective strategies to support participant retention.

organisations (POs) are also closely collaborating with scientific societies, for example the US Cystic Fibrosis Foundation, Cystic Fibrosis Therapeutics Development Network and European Cystic Fibrosis Society Clinical Trials Network. Another example is the Scleroderma Patient-centred Intervention Network, which is actively collecting real-world evidence. ${ }^{16}$ There is also a drive (via committees within the European Medicines Agency (EMA) such as the Pharmacovigilance Risk Assessment Committee, the Committee for Orphan Medicinal Products and the Paediatric Committee) to involve patients in European Union projects; for example, the Innovative Medicine Initiative (IMI)'s PARADIGM and PREFER projects seek to provide a framework for patient engagement. ${ }^{17} 18$ European Reference Networks on rare and complex diseases demonstrate that patients are crucial and considered as equal stakeholders in developing clinical practice guidelines and patient pathways. ${ }^{19}$ Greater involvement of patients in the clinical development process can lessen some challenges faced by the pharmaceutical industry and academia in conducting clinical trials, especially in rare diseases (box 1) ${ }^{20-23}$ Moreover, they help improve patient satisfaction with clinical trials, and aid development of new medicines to meet patient needs and expectations.

Different terms are used to describe the focus and collaboration of the pharmaceutical industry and academia with patients and POs, including patient involvement, patient engagement, patient-centricity and patient focus. In this article, 'patient focus' is used as an all-encompassing term, with the understanding that initiatives are designed and optimised to meet patient and caregiver needs and expectations. Here, we refer to 'patients', but recognise that many patient communities prefer the term 'people living with [a disease]'.

The approach to collaborations between research sponsors and the patient community varies widely, yet the potential benefits of such collaborations are recognised (eg, identification of unmet needs, determining the impact of new treatments, facilitating adherence, and improving trial design to enhance recruitment/ retention). ${ }^{2} 32124-27$ In their recent report, The Economist's Health Intelligence Unit emphasised the value of patient collaboration in expediting enrolment times for clinical trials (particularly in rare diseases) and increasing the likelihood of launch for new medicines. ${ }^{28}$ Supple et al provide guidance regarding collaboration with patients on IMI-funded European Union projects, such as U-BIOPRED,${ }^{29}$ and there are guidelines for patient engagement during the drug development life cycle. ${ }^{27} 3031$ However, there is a paucity of shared best practices and no practical framework to guide collaborations between the patient community and research sponsors, nor research evidence base evaluating the impact of collaborations. ${ }^{321}$

Here, we describe the establishment of a community advisory board $(\mathrm{CAB})$ : a transparent, multiyear collaboration between the scleroderma patient community and a clinical research sponsor (CRS). Since scleroderma, or systemic sclerosis, is a rare disease, the $\mathrm{CAB}$ was established following signing of the European Organisation for Rare Diseases (EURORDIS) charter. We present shared learnings from the collaboration and initiatives undertaken regarding the SENSCIS phase III clinical trial (NCT02597933) in scleroderma-associated interstitial lung disease (SSc-ILD) ${ }^{32}$ and initiatives not related to the trial. It is expected that these learnings will serve as a blueprint to guide and encourage future collaborations between patient communities, CRSs and other healthcare stakeholders, such as hospital-based investigators.

\section{DISEASE AND TRIAL OVERVIEW}

Scleroderma is a rare autoimmune disease that is characterised by the thickening and scarring of the skin and connective tissue of multiple organs in the body. It affects women more often than men and most commonly occurs between the ages of 30 and 50 . Scleroderma symptoms vary, depending on which parts of the body are affected, and can change over time. In some people, scleroderma affects only the skin. But in many people, scleroderma also harms structures beyond the skin, such as blood vessels and internal organs. The SENSCIS clinical trial investigated the efficacy and safety of nintedanib in patients with SSc-ILD, a common lung manifestation of scleroderma. A total of 576 patients with SSc-ILD aged $\geq 18$ years and with the onset of active disease $\leq 7$ years before screening were included and treated. The mean age of trial participants was 54 years and $75 \%$ were female.

\section{TRANSPARENCY AND DISCLOSURE}

In their publicly available charter, EURORDIS formalises the shared expectations of both patients and CRSs as 'the rapid acquisition of quality scientific data and the development of effective and safe treatments and the subsequent access to them' ${ }^{20}$ The EURORDIS charter outlines a framework for collaborations between POs and CRSs based on 11 key principles (box 2) ${ }^{20}$ The recognition of mutual interests and equal standing as stakeholders in 
Box 2 Principles of the European Organisation for Rare

Diseases Charter for Collaboration in Clinical Research

- This Charter is an expression of mutual intentions and aspirations.

- The Charter is not legally binding.

- The collaboration is based on respect and is not tokenistic.

- The CAB is recognised as an independent body and is not structurally dependent on the sponsor.

- The work and the structure are transparent.

- Agendas are cooperatively designed.

- The dialogue is meaningful and of high quality.

8. Collaboration between the sponsors and the CAB is timely where input can make a difference.

- Confidentiality is respected by both sides.

- The collaboration is based on trust.

All interactions are considered non-promotional.

$\mathrm{CAB}$, community advisory board.

clinical research provides a solid foundation for collaboration. It is also crucial to agree what is meant by terms such as 'patient', 'involvement' and 'key stakeholders'. ${ }^{21} 22$

Transparency is fundamental to establishing trust between stakeholders and sustaining a meaningful collaboration that acts with integrity. Some collaborations between POs and research sponsors have been subject to criticism and increased scrutiny by the medical community in recent years, ${ }^{33} 34$ due to a lack of transparency about these relationships and their funding. ${ }^{33-36}$ However, since 2012, members of the European Federation of Pharmaceutical Industries and Associations (EFPIA) and the Association of the British Pharmaceutical Industry are required to disclose all payments to POs. ${ }^{37}{ }^{38}$ Compliant with these guidelines, all funding and payments-in-kind were openly reported and are readily accessible to the public (see conflict of interests statement). This transparency also ensures that HTA bodies, payers and regulatory agencies can assess potential conflicts of interest of POs who may provide advice to them. For instance, the EMA has a policy in place stating patient representatives must declare any conflicts of interest; moreover, involvement with the EMA may be restricted if one's activities are felt to affect their impartiality. ${ }^{39}$

\section{ESTABLISHING A COMMUNITY ADVISORY BOARD}

The $\mathrm{CAB}$, comprising 10 delegates at initiation (rising to 15 over the course of the initiative) representing scleroderma POs from 10 countries, was established as the basis of the collaboration between the scleroderma patient community and the CRS. The first CAB was held after finalisation of the trial protocol but before training of investigators and initiation of the study. The demographics of the trial were generally reflected in the make-up of the $\mathrm{CAB}$, although the duration of active disease varied (making $\mathrm{CAB}$ members living with scleroderma not eligible for the trial). A CAB is a group of patients who offer their expertise to CRSs to discuss
Memorandum of Understanding

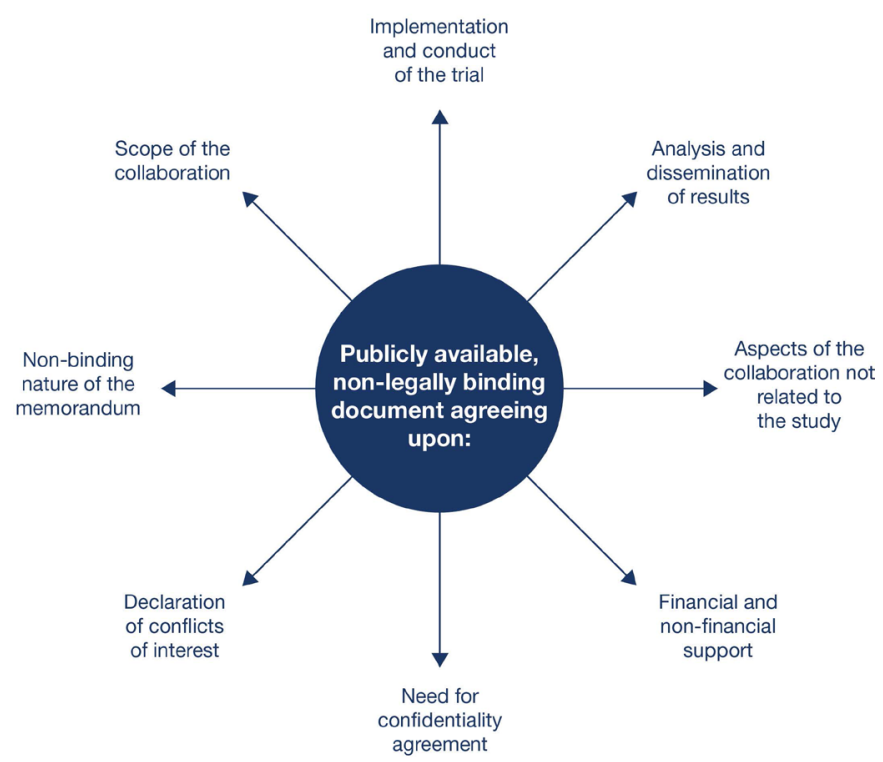

Figure 1 Areas covered by the memorandum of understanding between the research sponsor and scleroderma patient organisations.

* Signed by Canadian and European Scleroderma patient organisations.

overall programme development, a single trial or other projects beyond the research programme. Generally, the same group of patients advise several sponsors in their field. Ethically, it was important that the collaboration was based on a mutually accepted framework, allowed patients to contribute to an optimised patient-centric trial with suggested changes still subject to review board approval, and that $\mathrm{CAB}$ participants did not participate in the trial.

In accordance with the EURORDIS charter and its principles, the scope and terms of the collaboration between the scleroderma community and the CRS were laid out in a co-created, mutually ratified memorandum of understanding (MoU) (figure 1) ${ }^{40}$ prepared in accordance with the CRS's own Guiding Principles in the Collaboration with Patient Organisations. ${ }^{41}$ While the MoU is specific to the collaboration between the scleroderma POs and the CRS, the scleroderma $\mathrm{CAB}$ is open to other trial sponsors.

\section{FOUNDATIONS OF THE COLLABORATION}

From the CRS's perspective, the collaboration was founded on respect, trust, empathy and passion, and guided by the sponsor's code of conduct and principles for collaboration with POs. ${ }^{41}$ From the POs' perspective, the Federation of European Scleroderma Associations (FESCA), Scleroderma Canada and the Scleroderma Foundation share a common mission to represent the voice and interests of people living with scleroderma, their families and caregivers. POs (and representatives) approached the collaboration within their collective (and individual) remit to support people with scleroderma 
by promoting the advancement of knowledge, research and information in the field and increase awareness of the disease. As part of this, they encourage and/or undertake surveys, research projects and clinical trials related to the disease and the publication of the results of any such research.

Clear documentation of the CAB's advice and agreement on action items, agile working approaches, accountability and intrapreneurship helped to continuously optimise the CRS's initiatives to meet the needs and expectation identified by the CAB. Working methods included constant challenging of the status quo and optimisation through patient-focused feedback cycles (including user experience (UX)). The CAB identified challenges from the patient perspective; suggested and developed approaches to solve these challenges (including identification of existing solutions); concepts were tested; and existing solutions were adapted or new solutions were developed and optimised through testing and retesting with the $\mathrm{CAB}$ to address the challenges. The aim of the feedback cycles was to ensure that patient needs and patient satisfaction with trials and services were being met.

The following sections will provide an overview and the outcomes of the collaboration in three areas described in the MoU: the implementation and conduct of the clinical trial; analysis and dissemination of the results; and aspects of the collaboration not related to the trial.

\section{IMPLEMENTATION AND CONDUCT OF THE CLINICAL TRIAL}

The scleroderma CAB reviewed and provided advice on trial conduct and reporting to improve participant experience and trial retention. Figure 2 outlines the process by which the $\mathrm{CAB}$ provided advice, and how this was implemented in an iterative fashion, from initial advice to advice during conception and final advice. Each led

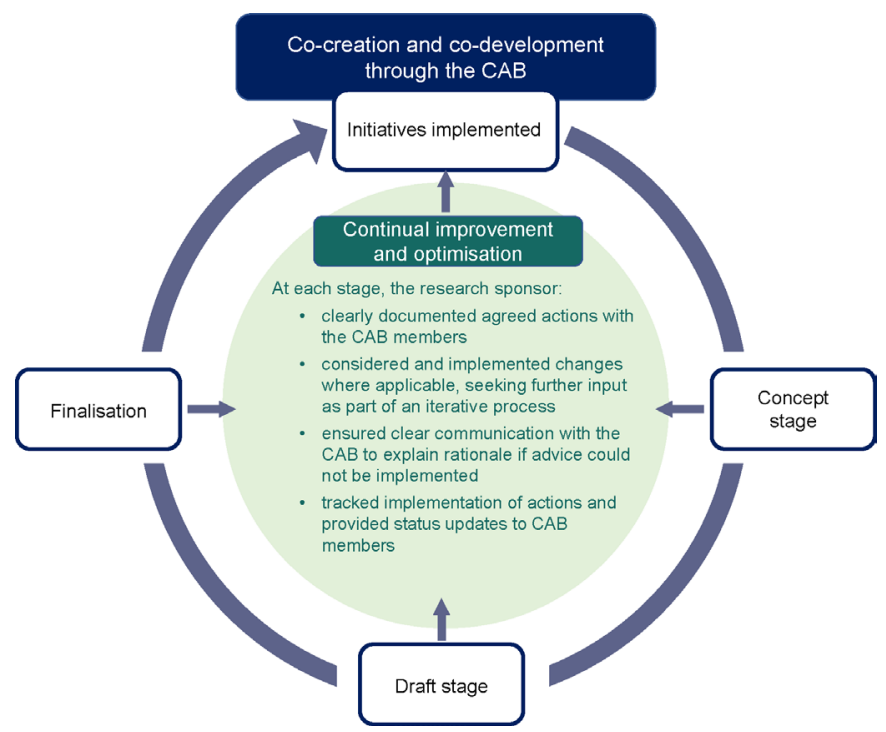

Figure 2 Process for receiving and implementing $C A B$ advice. CAB, community advisory board.

\section{Practical changes based on community advisory board feedback}

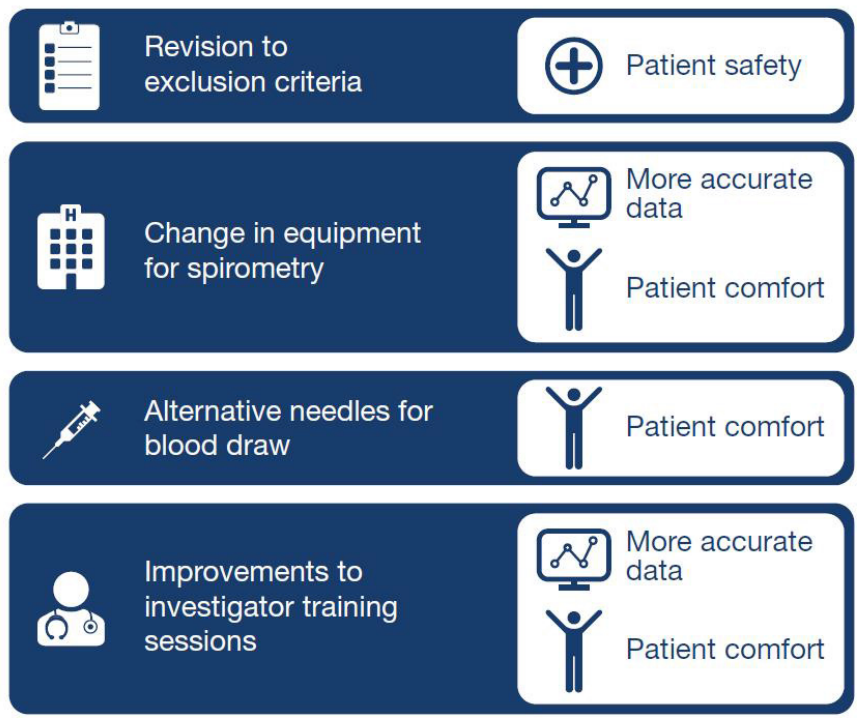

Figure 3 Patient-focused adaptations to the sclerodermaassociated interstitial lung disease trial based on the community advisory board's advice.

to the improvement and optimisation of trial procedures, and meaningful, patient-focused adaptations were made to the trial to address disease-specific challenges relevant to SSc-ILD patients (figure 3). All advised actions were clearly documented by the CRS and agreed with members of the CAB.

Initial input in the trial protocol from the scleroderma $\mathrm{CAB}$ facilitated revision of trial exclusion criteria. In response to insights on ulcerative manifestations, the exclusion criteria were revised so that patients with severe ulcers at anatomical sites other than their digits could also be excluded (at the discretion of the investigator) to ensure patient safety. Reviewing the protocol flowchart also led to practical adaptations to trial procedures and materials. Patients had reported discomfort and difficulties in forming a seal around the rigid, adult spirometry mouthpieces provided. In response to this feedback, paediatric silicone mouthpieces were provided to improve patient comfort and accuracy of pulmonary function tests. Patients also reported difficulties with blood-draw using conventional needles, so butterfly/ paediatric needles were provided to reduce discomfort.

Another area in which the CAB provided meaningful advice was regarding the modified Rodnan's skin score test, which was used as a secondary endpoint in the trial to assess skin thickening. Training in this outcome measure was provided to trial investigators, and the $\mathrm{CAB}$ suggested volunteers and provided advice on trainingsession conduct based on their experiences, notably the importance of room temperature and available dressing facilities. Due to skin fibrosis and reduced blood flow to the skin surface associated with Raynaud's phenomenon, people living with scleroderma are prone to the 
debilitating effects of cold temperatures ${ }^{42}{ }^{43}$; therefore, warmer room temperatures were provided to ensure the comfort of patients volunteering in expert training sessions. Anecdotal feedback from patients who volunteered for these sessions was positive, as their well-being had been sensitively accommodated, with many volunteers to participate in further training sessions as new investigator sites and countries onboarded. No patients discontinued participation of the sessions.

To keep trial participants informed and engaged throughout the trial, patient newsletters, birthday cards and thank-you letters were suggested and co-developed by $\mathrm{CAB}$ members and provided to trial participants through trial sites (approved by relevant ethics committees). Newsletters provided information about the status of the trial and regarding management of adverse events and challenges associated with scleroderma, such as digital ulcers and cold weather. The CAB felt that this information may have helped overcome 'participant burnout', and the trial had retention rates of more than $90 \% .^{32}$ Evidently, many factors feed into patient retention in trials that may be independent of the $\mathrm{CAB}$ input, such as those driven by investigator and hospital staff. It is impossible to be certain if this high retention rate is directly related to the $\mathrm{CAB}$ activities, but we would suspect some of it is. The CRS also sought the advice and feedback of the CAB on the wording and content of a post-trial survey, and iteratively incorporated changes where relevant.

\section{ANALYSIS AND DISSEMINATION OF RESULTS}

The contribution of $\mathrm{CAB}$ members to the clinical trial was acknowledged in the publication of the findings. ${ }^{32} \mathrm{CAB}$ advice regarding potential benefit/risk was sought prior to and after the results of the trial became available, and were included in the regulatory submission dossier of the trial sponsor.

To ensure that results of the trial were accessible to lay audiences and patients as per European Union clinical trial regulation, ${ }^{44}$ written lay summaries were developed by the trial sponsor with valuable input from the $\mathrm{CAB}$ to ensure that language and figures were understandable. ${ }^{45}$ In addition, and following $\mathrm{CAB}$ advice, the lay summary was developed in video format with input from the scleroderma $\mathrm{CAB}^{46}$

\section{ASPECTS OF THE COLLABORATION NOT RELATED TO THE TRIAL}

In parallel to the trial, the $\mathrm{CAB}$ and the CRS collaborated to co-develop opening tools for medication blister packs and bottles. This was because people living with scleroderma may find it difficult and painful to open their required medication blister packs and bottles, as scleroderma affects patients' hands, causing contractures of the fingers, shortening of the digits and digital ulcers. The co-development followed an interactive and iterative process over multiple meetings. The 3D-print design files of the blister-pack opening aid are publicly accessible ${ }^{4748}$
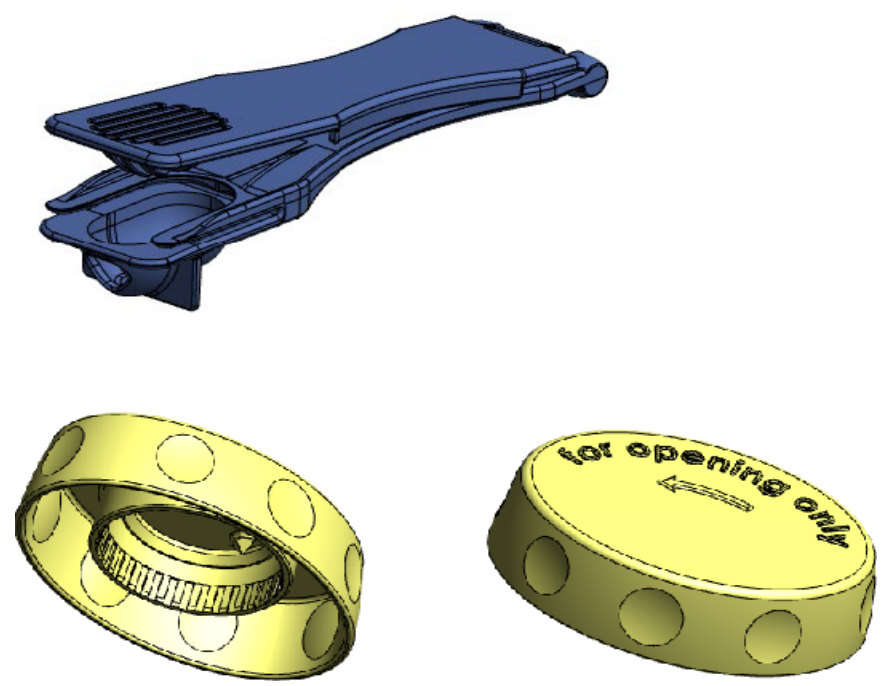

Figure 4 3D design images of the medication opening tools for bottles and blister packs.

in order to allow production and modification for blister packs of different sizes (figure 4). It is intended that the medication-opening tools and accompanying information leaflets will be made available to patients living with scleroderma to facilitate self-management of care.

To raise disease awareness amongst physicians, patients and their caregivers, educational materials to improve diagnosis and management of scleroderma were co-created and delivered by the scleroderma $\mathrm{CAB}$ and CRS, including comprehensive UX testing. Importantly, the research sponsor consulted with the $\mathrm{CAB}$ to determine what materials were already available regarding disease awareness and then adapted some materials for reuse. These included the SO RARE information cards (figure 5) intended for physicians and the general public, based on a disease awareness card originally designed by FESCA. Strategies to raise disease awareness culminated in the More than scleroderma health-literacy campaign, providing brochures and a publicly available online repository of information for people living with scleroderma and their caregivers. Three of the brochures from this campaign were recognised at the 2019 British Medical Association Patient Information Awards. The More than scleroderma online and printed materials aimed to widen access to high-quality, patient-focused and medically accurate information to raise disease awareness and improve health literacy among scleroderma patients, their physicians and caregivers. ${ }^{21} 22$

\section{LEARNINGS FOR THE FUTURE}

Many of the patient involvement initiatives undertaken within the collaboration are hoped to deliver a longterm legacy beyond trial completion, for the broader benefit of people living with scleroderma. In a changing digital and social environment, in which patients are more involved in their own medical care, collaboration 

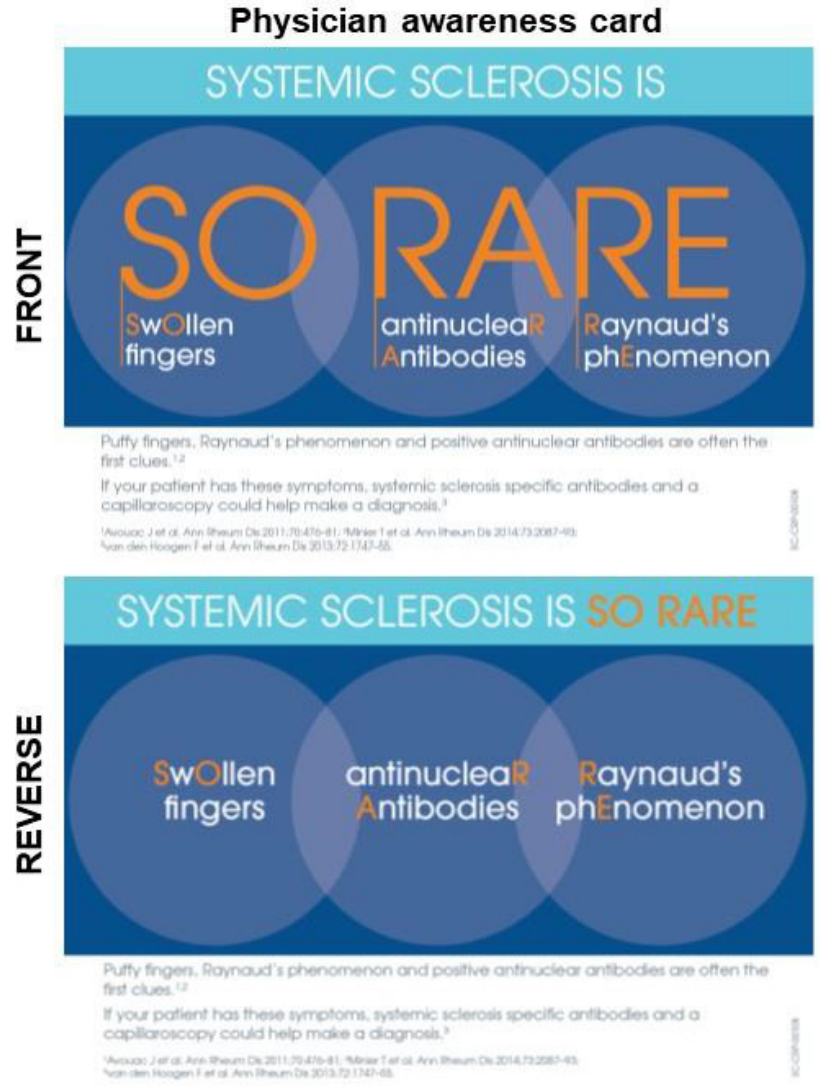

Patient awareness card

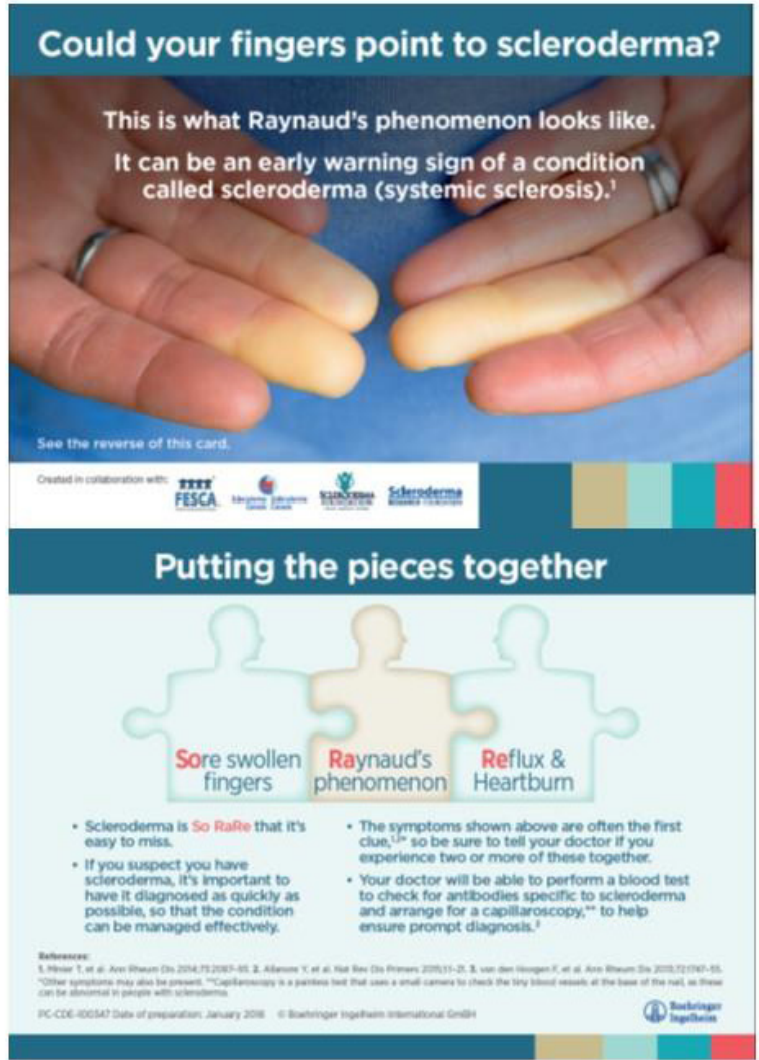

Figure 5 SO RARE disease awareness cards designed for physicians and patients. The image of hands used in the SO RARE cards is an Alamy Stock Photo, licensed for use until November 2022 (reference OY20206909)).

between patients, investigators and research sponsors is increasingly important.

Although the above modifications to trial design and conduct were hugely effective, the $\mathrm{CAB}$ and the research sponsor acknowledge that earlier $\mathrm{CAB}$ input during protocol design may have enabled inclusion of additional patient-focused measures to further improve trial participant experience. Although exploration for patient representation involvement was before the submission of the protocol, the first $\mathrm{CAB}$ was held after finalisation of the trial protocol but before training of the investigators and initiation of the study. Feedback on the protocol was, therefore, implemented through an amendment. Patient involvement for a dedicated study should start early in protocol development, and early enough to have their input during feasibility to allow for advice on patient materials as part of primary submission packages. Notably, earlier collaboration would have enabled access to the medication opening tool at the start of the trial. In future, it may be preferable to design medicine packaging that would not require such tools. Furthermore, it is difficult to assess the exact impact that patient advocacy can have on clinical research as we cannot always directly compare trials with and without patient input. Adequate methods to evaluate the impact of patients' advocacy are still needed.
Collaborations between POs and CRSs are necessary to confirm the unmet needs of patients and their caregivers, particularly in the field of rare diseases where specific needs can be overlooked. The collaboration benefited from already established POs. Initiatives such as OrphaNet and EURORDIS have large databanks that allow identification of existing POs in rare disease fields. Centres of expertise can be an alternative source to help identify and establish collaborations with rare disease patients in disease areas where established POs do not exist. The timing of patient involvement activities is critical to their success and impact. ${ }^{21}$ Although sometimes challenging to incorporate, initiatives should be timed for maximum effect across the entire life cycle of healthcare innovations. As the primacy of the patient voice becomes a key driving force for CRSs (and the wider pharmaceutical industry), it is imperative that long-term collaborations with POs are established and sustained beyond the confines of the clinical trial landscape alone. Through ongoing, transparent collaborations, POs and CRSs can collaborate to deliver meaningful, patient-focused outcomes encompassing the broader context of health and care provision needs.

\section{CONCLUSIONS}

This collaboration between POs and a CRS, facilitated through a $\mathrm{CAB}$ in a rare disease condition, led to 
meaningful improvements in patient safety, comfort, administration and self-management of care and addressed information needs (online supplemental file 1). Collaborations between the patient community and research sponsors must be guided by transparency, mutual interest, shared values, agreed codes of conduct, and ratified terms of engagement for PO and industry collaborations. The collaboration must be underpinned by the willingness of research sponsors to learn and optimise initiatives to meet patient and caregiver needs and expectations. The collaboration outlined here may serve as a template of best practice for future collaborations between POs, research sponsors and other healthcare stakeholders.

\section{Author affiliations}

${ }^{1}$ Federation of European Scleroderma Associations aisbl, Saint-Maur, Belgium

${ }^{2}$ Sklerodermiforening, Rødovre, Denmark

${ }^{3}$ Scleroderma Canada, Hamilton, Ontario, Canada

${ }^{4}$ Scleroderma Foundation, Inc, Danvers, Massachusetts, USA

${ }^{5}$ Gruppo Italiano per la Lotta alla Sclerodermia, Milan, Italy

${ }^{6}$ Scleroderma and Raynaud's UK, London, UK

${ }^{7}$ Portuguese Association of Patients with Scleroderma, Monção, Portugal

${ }^{8}$ Scleroderma Research Foundation, San Francisco, California, USA

${ }^{9}$ Asociación Española de Esclerodermia, Madrid, Spain

${ }^{10}$ EURORDIS-Rare Diseases Europe, Barcelona, Spain

${ }^{11}$ AK Gilbert Ltd, Brighton, UK

${ }^{12}$ Boehringer Ingelheim Schweiz GmbH, Basel, BS, Switzerland

${ }^{13}$ Boehringer Ingelheim International GmbH, Ingelheim am Rhein, Rheinland-Pfalz, Germany

${ }^{14}$ Boehringer Ingelheim Pharma GmbH and Co KG Biberach, Biberach, BadenWürttemberg, Germany

Acknowledgements The authors would like to thank Michael Oeschger (Sklerodermie Selbsthilfe e.V., Heilbronn, Germany) for his valuable contribution and commitment to advisory boards throughout the collaboration described below. The authors also acknowledge the contributions of Sylvia Franz, Kamila Sroka-Saidi and Hannah Delaney, all of Boehringer Ingelheim.

Contributors All authors made substantial and equal contributions to the conception and design of the work. AR, MS, EB, IG, AG, APPG, ATK, CL, RJR, JW, FH and $R C$ are nominated representatives for their respective national and international scleroderma patient organisations (as indicated in the affiliations). AG is a patient advocacy relations consultant engaged by the research sponsor (Boehringer Ingelheim International GmbH, Ingelheim am Rhein, Germany). MG, LM, JLLF, FS, WS and HF are paid employees of the research sponsor (Boehringer Ingelheim). The views expressed are those of the authors, and not necessarily those of their affiliated organisations.

Funding Under the authors' conceptual direction and feedback, editorial assistance (provided by Claire Scofield and Caroline Sproat of MediTech Media) was funded by Boehringer Ingelheim International $\mathrm{GmbH}$ (no grant/award number).

Competing interests The authors have read and understood the BMJ policy on declaration of interests and have the following interests to declare: AR, MS, JW, RJR, ATK, IG, EB, CL, AGo, APPG, FH and RC did not receive any personal payments for their contributions to this article. AR, MS, JW, RJR, ATK, IG, EB, CL and APPG declare payments from Boehringer Ingelheim International $\mathrm{GmbH}$ to their respective POs (FESCA aisbl., Scleroderma Canada and Scleroderma Foundation, USA) for participation in community advisory board meetings and payment of travel expenses. AGo declares that Boehringer Ingelheim International GmbH paid for their travel expenses for participation in community advisory board meetings. AR, MS, JW and RJR declare that their POs (FESCA aisbl., Scleroderma Canada and Scleroderma Foundation, USA) received additional payments from Boehringer Ingelheim International $\mathrm{GmbH}$ for speaker and advisory services including payment of travel expenses. FH and RC declare that Boehringer Ingelheim International $\mathrm{GmbH}$ paid for their travel expenses for speaker and advisory services including one $\mathrm{Bl}$ internal meeting and community advisory board meetings. RJR declares that the Scleroderma Foundation (USA) has received sponsorships in support of the annual scleroderma awareness raising campaign and the Scleroderma National Patient Education Conference from both Boehringer Ingelheim International GmbH and Boehringer Ingelheim Pharmaceuticals. FH and RC declare that EURORDISRare Diseases Europe has received sponsorships from Boehringer Ingelheim International GmbH for the EURORDIS Round Table of Companies and the European Conference on Rare Diseases and Orphan Products. AR and ATK declare that FESCA aisbl. has received sponsorships in support of their annual scleroderma awareness raising campaign and World Scleroderma Conference from Boehringer Ingelheim International GmbH. MS declares that Scleroderma Canada has received sponsorships in support of their Scleroderma Canada Patient Conference from Boehringer Ingelheim International $\mathrm{GmbH}$. These sponsorships are not related to this article. AGi has worked with the research sponsor (BI) as a paid consultant since September 2016. MG, LM, JLLF, FS, WS and HF are paid employees of the research sponsor (BI).

Patient and public involvement This article is co-authored by patient representatives, testament to the equal standing shared by the European Organisation for Rare Diseases (EURORDIS), scleroderma patient organisations and the clinical research sponsor, who formed this collaboration. By mutual agreement, a scleroderma patient organisation representative is lead author in recognition of their contribution to the collaboration, the primacy of patients as key stakeholders in clinical research, and their integral role in establishing effective collaborations with research sponsors.

Patient consent for publication Not required.

Provenance and peer review Not commissioned; externally peer reviewed.

Supplemental material This content has been supplied by the author(s). It has not been vetted by BMJ Publishing Group Limited (BMJ) and may not have been peer-reviewed. Any opinions or recommendations discussed are solely those of the author(s) and are not endorsed by BMJ. BMJ disclaims all liability and responsibility arising from any reliance placed on the content. Where the content includes any translated material, BMJ does not warrant the accuracy and reliability of the translations (including but not limited to local regulations, clinical guidelines, terminology, drug names and drug dosages), and is not responsible for any error and/or omissions arising from translation and adaptation or otherwise.

Open access This is an open access article distributed in accordance with the Creative Commons Attribution Non Commercial (CC BY-NC 4.0) license, which permits others to distribute, remix, adapt, build upon this work non-commercially, and license their derivative works on different terms, provided the original work is properly cited, appropriate credit is given, any changes made indicated, and the use is non-commercial. See: http://creativecommons.org/licenses/by-nc/4.0/.

ORCID iD

Joep Welling http://orcid.org/0000-0002-2179-2110

\section{REFERENCES}

1 Holm KE, Casaburi R, Cerreta S, et al. Patient involvement in the design of a patient-centered clinical trial to promote adherence to supplemental oxygen therapy in COPD. Patient 2016;9:271-9.

2 Price A, Albarqouni L, Kirkpatrick J, et al. Patient and public involvement in the design of clinical trials: an overview of systematic reviews. J Eval Clin Pract 2018;24:240-53.

3 Pushparajah DS. Making patient engagement a reality. Patient 2018;11:1-8.

4 US Food \& Drug Administration. CDER patient-focused drug development, 2019. Available: https://www.fda.gov/drugs/ development-approval-process-drugs/cder-patient-focused-drugdevelopment [Accessed 5 Dec 2019].

5 European Medicines Agency. EMA and FDA reinforce collaboration on patient engagement, 2016. Available: https://www.ema.europa. eu/en/news/ema-fda-reinforce-collaboration-patient-engagement [Accessed 5 Dec 2019].

6 Cabinet Office (UK). Medicines, medical devices and blood regulation and safety: marketing authorisations, variations and licensing guidance, 2019. Available: https://www.gov.uk/topic/medicinesmedical-devices-blood/marketing-authorisations-variations-licensing [Accessed 5 Dec 2019].

7 National Institute for Health and Care Excellence. Making a positive impact, 2019. Available: https://www.nice.org.uk/about/nicecommunities/nice-and-the-public/public-involvement/making-apositive-impact [Accessed 5 Dec 2019].

8 National Institute for Health and Care Excellence. Guide to the processes of technology appraisal, 2018. Available: https://www. 
nice.org.uk/Media/Default/About/what-we-do/NICE-guidance/NICEtechnology-appraisals/technology-appraisal-processes-guide-apr2018.pdf [Accessed 5 Dec 2019].

9 Scottish Medicines Consortium. Making a submission, 2019. Available: https://www.scottishmedicines.org.uk/making-asubmission [Accessed 5 Dec 2019].

10 European Network for Health Technology Assessment. Submission \& methodology, 2019. Available: https://eunethta.eu/methods-andprocedures/ [Accessed 5 Dec 2019].

11 Innovative Medicines Initiative. IMI mission and objectives, 2019. Available: https://www.imi.europa.eu/about-imi/mission-objectives [Accessed 5 Dec 2019].

12 Patient Focused Medicines Development. Integrating the voice of the patient across the lifecycle of medicine, 2019. Available: https://pati entfocusedmedicine.org/about-pfmd/ [Accessed 5 Dec 2019].

13 International Council for Harmonisation of Technical Requirements for Pharmaceuticals for Human Use. ICH Harmonised guideline: general considerations for clinical studies, 2019. Available: https:// www.ich.org/page/efficacy-guidelines [Accessed 5 Dec 2019].

14 European Patients' Academy. Guidance for patient involvement in industry-led medicines R\&D, 2016. Available: https://toolbox.eupati. eu/resources/guidance-for-patient-involvement-in-industry-ledmedicines-rd/ [Accessed 5 Dec 2019].

15 The Denver principles. Women Alive 2000;12.

16 Kwakkenbos L, Thombs BD, Khanna D, et al. Performance of the patient-reported outcomes measurement information System-29 in scleroderma: a scleroderma patient-centered intervention network cohort study. Rheumatology 2017;56:1302-11.

17 Innovative Medicines Initiative. Paradigm, 2019. Available: https:// www.imi.europa.eu/projects-results/project-factsheets/paradigm [Accessed 5 Dec 2019].

18 PREFER. Home page, 2019. Available: https://www.imi-prefer.eu [Accessed 5 Dec 2019].

19 Smith V, Scirè CA, Talarico R, et al. Systemic sclerosis: state of the art on clinical practice guidelines. RMD Open 2018;4:e000782.

20 EURORDIS. EURORDIS charter for collaboration in clinical research in rare diseases, 2009. Available: https://www.eurordis.org/content/ eurordis-charter-clinical-trials-rare-diseases [Accessed 5 Dec 2019].

21 Hoos A, Anderson J, Boutin M, et al. Partnering with patients in the development and lifecycle of medicines: a call for action. Ther Innov Regul Sci 2015;49:929-39.

22 Distler $\mathrm{O}$, Allanore $\mathrm{Y}$, Denton CP, et al. Factors influencing early referral, early diagnosis and management in patients with diffuse cutaneous systemic sclerosis. Rheumatology 2018;57:813-7.

23 Page SJ, Persch AC, Recruitment PAC. Recruitment, retention, and blinding in clinical trials. Am J Occup Ther 2013;67:154-61.

24 Thornton $\mathrm{H}$. Patient and public involvement in clinical trials. BMJ 2008;336:903-4.

25 Adams RJ. Improving health outcomes with better patient understanding and education. Risk Manag Healthc Policy 2010;3:61-72.

26 Tapp H, Derkowski D, Calvert M, et al. Patient perspectives on engagement in shared decision-making for asthma care. Fam Pract 2017;34:353-7.

27 Stein S, Bogard E, Boice N, et al. Principles for interactions with biopharmaceutical companies: the development of guidelines for patient advocacy organizations in the field of rare diseases. Orphanet $J$ Rare Dis 2018;13:18.

28 The Economist Intelligence Unit. The innovation imperative: the future of drug development. Part I: research methods and findings, 2018. Available: https://druginnovation.eiu.com/research-methodsand-findings/ [Accessed 10 Jan 2020].

29 Supple D, Roberts A, Hudson V, et al. From tokenism to meaningful engagement: best practices in patient involvement in an EU project. Res Involv Engagem 2015;1:5.
30 Hunter A, Facey K, Thomas V, et al. EUPATI guidance for patient involvement in medicines research and development: health technology assessment. Front Med 2018;5:270.

31 Kirwan JR, de Wit M, Frank L, et al. Emerging guidelines for patient engagement in research. Value Health 2017;20:481-6.

32 Distler O, Highland KB, Gahlemann M, et al. Nintedanib for systemic sclerosis-associated interstitial lung disease. N Engl J Med 2019;380:2518-28.

33 McCoy MS, Carniol M, Chockley K, et al. Conflicts of interest for patient-advocacy organizations. N Engl J Med 2017;376:880-5.

34 Ozieranski P, Rickard E, Mulinari S. Exposing drug industry funding of UK patient organisations. BMJ 2019;365:11806.

35 Moberly T. NHS joint working with industry is out of public sight. BMJ 2019;364:11353.

36 Salisbury H. Helen Salisbury: who let the drug companies in? BMJ 2019;365:11581.

37 efpia. Working together with patients, 2019. Available: https:// www.efpia.eu/media/413114/workingtogetherwithpatients_patientremuneration-principles.pdf

38 Association of the British Pharmaceutical Industry. Code of practice for the pharmaceutical industry 2019, 2019. Available: https://www. abpi.org.uk/media/6655/abpi-code-of-practice-2019.pdf

39 European Medicines Agency. Getting involved, 2019. Available: https://www.ema.europa.eu/en/partners-networks/patientsconsumers/getting-involved [Accessed 5 Dec 2019].

40 Boehringer Ingelheim International $\mathrm{GmbH}$, Federation of European Scleroderma Associations, The Scleroderma Society of Canada. Memorandum of understanding between Boehringer Ingelheim (BI), Federation of European Scleroderma Associations (FESCA), The Scleroderma Society of Canada (SSC). Regarding the SENSCIS ${ }^{\mathrm{TM}}$ trial, its extension trial and areas of collaboration not related to the trial (the 'Collaboration'), 2016. Available: https:// fesca-scleroderma.eu/wp-content/uploads/2019/02/Final_SSc MoU.pdf [Accessed 5 Dec 2019].

41 Boehringer Ingelheim. How we collaborate with patient organisations, 2018. Available: https://www.boehringer-ingelheim.com/ourcompany/corporate-citizenship/patient-organisations [Accessed 5 Dec 2019].

42 Pauling JD, Saketkoo LA, Matucci-Cerinic M, et al. The patient experience of Raynaud's phenomenon in systemic sclerosis. Rheumatology 2019;58:18-26.

43 Watson HR, Robb R, Belcher G, et al. Seasonal variation of Raynaud's phenomenon secondary to systemic sclerosis. $J$ Rheumatol 1999;26:1734-7.

44 European Commission. Summaries of clinical trial results for laypersons, 2017. Available: https://ec.europa.eu/health/sites/health/ files/files/eudralex/vol-10/2017_01_26_summaries of ct results for laypersons.pdf [Accessed 5 Dec 2019].

45 Boehringer Ingelheim $\mathrm{GmbH}$. A study to compare nintedanib with placebo for patients with scleroderma-related lung fibrosis (SENSCIS ${ }^{\circledR}$ study, 1199.214), 2019. Available: https://trials. boehringer-ingelheim.com/public/trial results documents/1199/ english_1199214laysummaryenglishpdf.pdf\#page $=1$ [Accessed $\overline{5}$ Dec 2019].

46 Boehringer Ingelheim $\mathrm{GmbH}$. SENSCIS ${ }^{\circledR}$ : a study to compare nintedanib with placebo for patients with scleroderma-related lung fibrosis, 2019. Available: https://players.brightcove.net/ 4309680634001/default default/index.html?videold=6060301840001 [Accessed 5 Dec 2019].

47 YouMagine. Blister pack opener, 2019. Available: https://www. youmagine.com/designs/blister-pack-opener\#.W22EYxuV49A [Accessed 5 Dec 2019].

48 Thingiverse. Blister pack opener, 2018. Available: https://www. thingiverse.com/thing:3080416 [Accessed 5 Dec 2019]. 